\title{
Static and dynamic VaR constrained portfolios with application to delegated portfolio management
}

\section{Mustafa Ç. Pınar}

To cite this article: Mustafa Ç. Pınar (2013) Static and dynamic VaR constrained portfolios with application to delegated portfolio management, Optimization, 62:11, 1419-1432, DOI: 10.1080/02331934.2013.854785

To link to this article: http://dx.doi.org/10.1080/02331934.2013.854785

曲 Published online: 18 Nov 2013.

Submit your article to this journal

Џ Article views: 123

Q View related articles $₫$

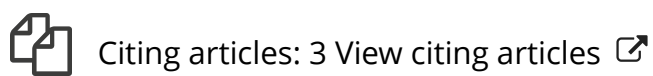




\title{
Static and dynamic VaR constrained portfolios with application to delegated portfolio management
}

\author{
Mustafa Ç. Pınar* \\ Department of Industrial Engineering, Bilkent University, Ankara, Turkey
}

(Received 16 January 2013; accepted 9 October 2013)

\begin{abstract}
We give a closed-form solution to the single-period portfolio selection problem with a Value-at-Risk $(\mathrm{VaR})$ constraint in the presence of a set of risky assets with multivariate normally distributed returns and the risk-less account, without short sales restrictions. The result allows to obtain a very simple, myopic dynamic portfolio policy in the multiple period version of the problem. We also consider mean-variance portfolios under a probabilistic chance (VaR) constraint and give an explicit solution. We use this solution to calculate explicitly the bonus of a portfolio manager to include a VaR constraint in his/her portfolio optimization, which we refer to as the price of a VaR constraint.
\end{abstract}

Keywords: dynamic portfolio selection; probabilistic chance constraint; value-at-risk; mean-variance efficient portfolios; delegated portfolio management

AMS Subject Classifications: 91G10; 91B30; 90C90

\section{Introduction}

The problem of selecting an optimal portfolio is a central problem in finance. The MeanVariance portfolio theory introduced by Markowitz [1] had a tremendous impact on the development of financial mathematics; see [2] for a more recent review. The Markowitz framework advocated selecting a portfolio minimizing risk measured by the variance of portfolio return while aiming for a minimum target return or maximizing expected return while controlling the variance of the portfolio return. While not as popular as the MeanVariance portfolio theory, there exists other approaches to the optimal portfolio choice, e.g. expected utility maximization, probabilistic chance constraints and so on; the literature is vast on all these topics, for a sample see e.g. [2-6]. The Value-at-Risk introduced by Jorion [7] is a widely used measure of risk in finance. For a given financial portfolio and a selected probability level it gives the threshold value such that the loss of the portfolio exceeds that threshold value with the given probability level. Imposing a limit on the Value-at-Risk thus involves a probabilistic chance constraint. Although chance constrained portfolio selection problems and second-order cone programming problems with a single cone constraint have been studied previously,[8-11] it appears that a simple, closed-form solution in the case of normally distributed returns of risky assets and no short sales restrictions has not been

*Email: mustafap@bilkent.edu.tr 
available, to the best of the author's knowledge. Partial explanations for the absence of closed-form solutions could be the advent of very efficient algorithms and software for conic convex optimization problems, which made possible the treatment of portfolio problems with more general restrictions such as no-short sales. The only partial exception to this lack of interest in explicit solutions is the textbook by Ruszczynski [12] where a similar problem is set-up and solved in closed-form in an exercise without a budget constraint and excluding the risk-less asset. The author does not elaborate on the solution, does not allow short positions and does not study a dynamic problem. Interestingly, the book,[13] while published later and discussing at length the portfolio selection problem with probabilistic chance constraints, ignores the solution in [12]. In the light of these remarks, the first main contribution of this paper is to prove closed-form portfolio results using convex (conic) duality theory (see $[9,12]$ for treatments of conic duality for second-order cone programming problems), and explore the consequences in a dynamic portfolio choice setting in Sections 2 and 3 when the investor has a risk neutral objective function. The second contribution is an explicit formula for the case of mean-variance portfolios under a chance constraint in Section 4. Admittedly, the multivariate normally distributed returns and absence of restrictions on short positions constitute unrealistic assumptions. However, the simplicity of the solutions obtained in the present paper helps deliver simple insights analytically about optimal portfolios, and also allows an explicit solution in a Delegated Portfolio Management model,[14-16] which is the third contribution of the paper. The Portfolio Delegation problem is described in Section 5. We consider a setting where an investor not willing or not able to invest on his/her own delegates investment to a portfolio manager by means of a contract which is an affine function of the wealth realized at the end of the horizon. We address the following problem: how much bonus should the investor pay to the portfolio manager in order to convince the manager to include a VaR constraint in optimizing the portfolio? We compute in closed form the bonus, and investigate its dependencies on problem parameters. We conclude the paper in Section 6 with a summary of results and future research directions. To keep with length restrictions we omit the proofs of the results. They can be accessed in the online technical report version of the paper.[17]

The results presented in the paper can also be positioned within the existing finance literature on VaR restricted portfolio choice problem. An early reference is Roy [18] where a safety first approach to a static portfolio problem is discussed under the assumption that only the first and second moments of asset returns are available. In particular, the case of normal distribution and continuous time (Brownian motion) is dealt with in Başak and Shapiro [19] using the methods of continuous time finance; see also the review and references therein. They consider a general utility function and establish that the solution to the VaR problem involves an option problem, whereby the payout over a certain range of the stock price realizations is enhanced by a corridor option (a corridor option is like a barrier option, where a range is specified for the reference instrument, and for every day that the instrument's value falls within the range, a pay-off is earned by the holder of the option), at the cost of an amount invested in the stock and risk-less bond. The solution presented in the present paper considers a proportional decrease in the stock investment with a simultaneous increase in the bond investment, since there are no derivative instruments traded in our setting. When risky returns are normally distributed, so that the markets can be viewed as complete and options exist, Başak and Shapiro show that the optimal solution is to have a limited increase in investment for a subset large enough to meet the VaR restriction (by means of options) and to cut down on the investment in the stocks and bond. See also 
Danielsson et al. [20] for an exposition in a discrete setting. Note also that the portfolio insurance problem as discussed in Grossman and Vila [21] is related to the VaR restricted portfolio optimization problems of the present paper since it corresponds to the case of the parameter $\alpha$ (see below) set equal to zero, i.e. the final portfolio value is not allowed to fall below a target.

\section{The setting and the single-period portfolio policy}

We wish to invest capital $W_{0}$ in $n+1$ assets, the first $n$ of which are risky assets and the last one represents a risk-free asset, e.g. the bank account. Each risky asset has a respective random rate of return $\mathcal{R}_{i}$ during the investment horizon for $i=1, \ldots, n$, and the riskfree asset the fixed rate $R$. We assume that the rates of return of risky assets collected in the random vector $\mathcal{R}$ follow a Normal distribution with mean $\mu$ and positive definite variance-covariance matrix $\Sigma$.

Let $x_{i}$ the monetary amount invested in the $i$-th asset. At the end of the horizon, the realized wealth $W_{1}$ is a random variable given by

$$
W_{1}=\sum_{i=1}^{n} \mathcal{R}_{i} x_{i}+x_{n+1} R .
$$

We are interested in the solution of the following problem:

$$
\begin{aligned}
& \max \mathbb{E}\left[W_{1}\right] \\
& \text { s.t. } \sum_{i=1}^{n+1} x_{i}=W_{0} \\
& \operatorname{Pr}\left\{W_{1} \geq b\right\} \geq 1-\alpha
\end{aligned}
$$

for some positive constant $\alpha \in(0,1]$ (the smaller $\alpha$ is, the more protection is enforced). The last constraint above is a probabilistic constraint, also known as a Chance Constraint. It expresses the requirement that the realized wealth at the end of the horizon exceed a certain target wealth $b$ with probability at least $1-\alpha$. By passing to a loss representation using $b-W_{1}$, the above constraint can be recast as a Value-at-Risk constraint as well; see p. 16 of [13] for details. A detailed discussion of Value-at-Risk in an optimization context can be found in [22].

Let us collect the portfolio positions in the risky assets in the $n$-vector $x$. It is easy to see using well-known techniques that the above problem is equivalent to the Chance Constrained Portfolio Problem (CCPP)

$$
\begin{aligned}
& \max \bar{\mu}^{T} x+W_{0} R \\
& \text { s.t. } z_{\alpha} \sqrt{x^{T} \Sigma x} \leq \bar{\mu}^{T} x-b+W_{0} R
\end{aligned}
$$

where $\bar{\mu}=\mu-R \mathbf{1}(\mathbf{1}$ denotes a $n$-vector of all ones $), z_{\alpha}:=\Phi^{-1}(1-\alpha)$ is the $(1-\alpha)$-quantile of the standard Normal distribution and $\Phi(z)$ is the cdf of a standard Normal random variate; c.f. [13]. If $\alpha$ satisfies $0<\alpha \leq 1 / 2$ then $z_{\alpha} \geq 0$ and the problem above is a convex conic (second-order cone) optimization problem. Variants of this problem have been formulated and solved in the context of portfolio optimization under various additional restrictions in the portfolio positions, see e.g. [23]. One usually resorts to available second-order cone 
optimization solvers to solve numerically the resulting portfolio problems. Second-order cone programming problems with a single cone constraint have been studied in e.g. [10] where an algorithm exploiting the presence of a single cone constraint is proposed for their numerical solution. The simple version of the problem formulated here can be solved analytically without resorting to an algorithm, a fact that seems to have gone undocumented thus far.

In the present paper, we shall first be concerned with closed-form solution of (CCPP) and its consequences in a multi-period setting.

\section{Proposition 1}

$$
\begin{aligned}
& \text { If } z_{\alpha}>\sqrt{\mathcal{H}} \text { and } b<W_{0} R \text { then }(C C P P) \text { admits an optimal solution given by } \\
& \qquad x^{*}=\left(\frac{W_{0} R-b}{z_{\alpha} \sqrt{\mathcal{H}}-\mathcal{H}}\right) \Sigma^{-1} \bar{\mu}
\end{aligned}
$$

where $\mathcal{H}=\bar{\mu}^{T} \Sigma^{-1} \bar{\mu}$.

(2) If $z_{\alpha}>\sqrt{\mathcal{H}}$ and $b>W_{0} R$ then (CCPP) is infeasible.

(3) If $z_{\alpha}<\sqrt{\mathcal{H}}$ and $b<W_{0} R$ then (CCPP) is unbounded.

Proposition 1 shows that a (CCPP) solving investor makes an optimal portfolio choice if he/she chooses a stringent probabilistic guarantee that is larger than the market optimal Sharpe ratio (the quantity $\sqrt{\mathcal{H}}$ that is known from MV portfolio theory as the slope of the Capital Market Line (see e.g. [24]) plays an important role in Proposition 1 as well as in subsequent sections), and a target wealth smaller than the wealth that would be obtained by putting all the initial wealth into the risk-less asset. Put in other words, strong protection (i.e. small probability of falling short of target wealth) coupled with a relatively low target results in an optimal portfolio rule while the combination of strong protection and high target does not give any feasible portfolio.

The only case not covered by the above result is when $z_{\alpha}<\sqrt{\mathcal{H}}$ and $b>W_{0} R$. In this case, although not strictly guaranteed, we expect (CCPP) to be unbounded. This happens if it is feasible since the dual is surely infeasible following part 3 of Proposition 1.

Note that the optimal portfolio of Proposition 1 is a mean-variance (MV) efficient portfolio. The optimal position in the risk-less asset is obtained as $W_{0}-\left[\frac{W_{0} R-b}{z_{\alpha} \sqrt{\mathcal{H}}-\mathcal{H}}\right] \mathbf{1}^{T} \Sigma^{-1} \bar{\mu}$. For the case $b=W_{0} R$ the optimal portfolio is a totally risk-less portfolio, i.e. all of initial wealth is invested into the risk-less asset. The optimal expected excess return $\bar{\mu}^{T} x^{*}=$ $\frac{W_{0} R \sqrt{\mathcal{H}}}{z_{\alpha}-\sqrt{\mathcal{H}}}-\frac{\sqrt{\mathcal{H}}}{z_{\alpha}-\sqrt{\mathcal{H}}} b$ depends linearly on target wealth $b$. In case 1 of the above result, the expected excess return increases with decreasing target wealth $b$. It is apparent from the form of the optimal portfolio that as the protection level increases (i.e. $z_{\alpha}$ increases) the portfolio tends to put more weight into the risk-less asset.

In the multi-period case we shall also be interested in a slight generalization of the CCPP which we shall refer to as (aCCPP):

$$
\begin{aligned}
& \max a \bar{\mu}^{T} x+W_{0} R \\
& \text { s.t. } z_{\alpha} \sqrt{x^{T} \Sigma x} \leq \bar{\mu}^{T} x-b+W_{0} R
\end{aligned}
$$


for some scalar $a$. We can state now the corresponding result to Proposition 1 for (aCCPP). The proof is similar to the proof of Proposition 1. We assume $b<W_{0} R$.

Proposition 2 If $a>0$ and $z_{\alpha}>\sqrt{\mathcal{H}}$ then (aCCPP) admits an optimal solution given by

$$
x^{*}=\left(\frac{W_{0} R-b}{z_{\alpha} \sqrt{\mathcal{H}}-\mathcal{H}}\right) \Sigma^{-1} \bar{\mu} .
$$

The result is unaffected by the choice of the positive scalar $a$.

\section{The multi-period VaR-constrained model}

We consider now a multi-period version of the portfolio choice problem. The investment horizon is divided into $N$ periods, in each of which the rates of return $\mathcal{R}_{i}, i=1, \ldots, n$ of risky assets are independently and identically (multivariate normally) distributed with mean vector $\mu_{t}$ and positive definite variance-covariance matrix $\Sigma_{t}, t=1, \ldots, N$. For simplicity, we assume that the risk-less account has return equal to $R$ throughout the horizon.

Given an initial wealth $W_{0}$ at the beginning of the investment horizon, i.e. beginning of time period $t=1$, minimum target levels $b_{t}$ for the chance constraints in each period, and appropriately chosen positive scalars $z_{\alpha, t}$ for $t=1, \ldots, N$, denoting the $n$-dimensional portfolio decision vectors in risky assets $x^{t}, t=1, \ldots, N$, the multi-period VaR-constrained portfolio selection problem is posed as follows:

$$
\begin{gathered}
V_{N}^{*}=\max _{x^{n} \in X_{N}} \bar{\mu}_{N}^{T} x^{N}+W_{N-1} R \\
V_{N-1}^{*}=\max _{x^{n-1} \in X_{n-1}} \mathbb{E}_{N-1}\left[V_{N}^{*}\right] \\
\vdots \\
V_{2}^{*}=\max _{x^{2} \in X_{2}} \mathbb{E}_{2}\left[V_{3}^{*}\right] \\
V_{1}^{*}=\max _{x^{1} \in X_{1}} \mathbb{E}\left[V_{2}^{*}\right]
\end{gathered}
$$

where $X_{t}=\left\{x \in \mathbb{R}^{n}: z_{\alpha, t} \sqrt{x^{T} \Sigma_{t} x} \leq \bar{\mu}_{t}^{T} x-b_{t}+W_{t-1} R\right\}, \bar{\mu}_{t}=\mu_{t}-R \mathbf{1}$, for $t=1, \ldots, N$, and $\mathbb{E}_{t}[$.] denotes expectation conditioned on the information known at the beginning of decision period $t$. We assume $b_{t}<W_{t-1} R$ for every $t=1, \ldots, N$.

We have the following result.

Proposition 3 Under the choices $z_{\alpha, t}>\sqrt{\mathcal{H}_{t}}$, where $\mathcal{H}_{t}=\bar{\mu}_{t}^{T} \Sigma_{t}^{-1} \bar{\mu}_{t}$, for $t=1, \ldots, N$ the dynamic portfolio policy given by

$$
x^{t *}=\left[\frac{\left(W_{t-1} R-b_{t}\right)}{z_{\alpha, t} \sqrt{\mathcal{H}_{t}}-\mathcal{H}_{t}}\right] \Sigma_{t}^{-1} \bar{\mu}_{t},
$$

with

$$
V_{t}^{*}=\sum_{j=t}^{N}\left(\prod_{i=j+1}^{N}\left(1-\gamma_{i} \mathcal{H}_{i}\right)\right) R^{N-j} b_{j} \gamma_{j} \mathcal{H}_{j}+\left(\prod_{i=t}^{N}\left(1-\gamma_{i} \mathcal{H}_{i}\right)\right) R^{N-(t-1)} W_{t-1}
$$


where $\gamma_{t}=\frac{1}{\mathcal{H}_{t}-z_{\alpha, t} \sqrt{\mathcal{H}_{t}}}$, for $t=1, \ldots, N$ solves the multi-period VaR-constrained portfolio selection problem.

The result implies that in the multi-period case, the optimal solution prescribes a myopic dynamic portfolio choice; see [25] for a discussion of myopic multi-period portfolio policies.

\section{Chance constrained mean-variance portfolios}

Now, we shall turn to the problem of selecting a portfolio according to the Mean-Variance criterion while satisfying a VaR-constraint as in the previous sections. Without repeating the details we pose the problem we refer to as (MVCCPP) directly as follows:

$$
\begin{aligned}
& \max \bar{\mu}^{T} x+W_{0} R-\frac{\rho}{2} x^{T} \Sigma x \\
& \text { s.t. } z_{\alpha} \sqrt{x^{T} \Sigma x} \leq \bar{\mu}^{T} x-b+W_{0} R
\end{aligned}
$$

where we introduced a positive scalar $\rho$, a parameter controlling the aversion to large variance of expected portfolio return. The above model gives two handles on risk control to the investor: in addition to ensuring that the probability of falling below a target wealth is small, it also penalizes large variations in expected portfolio return à la Markowitz. Now, we shall prove a result which completely characterizes the optimal solution of the problem (MVCCPP). We denote as usual by $\mathcal{H}$ the quantity $\bar{\mu}^{T} \Sigma^{-1} \bar{\mu}$.

Proposition 4

(A) If

(1) $z_{\alpha}>\sqrt{\mathcal{H}}, b<W_{0} R$ and $\rho<\frac{z_{\alpha} \sqrt{\mathcal{H}}-\mathcal{H}}{W_{0} R-b}$ or,

(2) $0<z_{\alpha}<\sqrt{\mathcal{H}}, b>W_{0} R$ and $\rho>\frac{z_{\alpha} \sqrt{\mathcal{H}}-\mathcal{H}}{W_{0} R-b}$

then (MVCCPP) admits an optimal solution given by

$$
x^{*}=\left(\frac{W_{0} R-b}{z_{\alpha} \sqrt{\mathcal{H}}-\mathcal{H}}\right) \Sigma^{-1} \bar{\mu} .
$$

(B) If $z_{\alpha}>0$, and $\rho \geq \frac{z_{\alpha} \sqrt{\mathcal{H}}-\mathcal{H}}{W_{0} R-b}$ (regardless of the choice of $\left.b\right)$ then (MVCCPP) admits an optimal solution given by

$$
x^{*}=\frac{1}{\rho} \Sigma^{-1} \bar{\mu} .
$$

(C) If $z_{\alpha}>\sqrt{\mathcal{H}}, b>W_{0} R$ then MVCCPP is infeasible.

Notice that in Part A, the optimal portfolio expression does not contain the variance aversion parameter $\rho$, and hence is only dependent on it indirectly. This dependence is through a critical value of $\rho$ which is equal to the inverse of the optimal portfolio constant $\frac{W_{0} R-b}{z_{\alpha} \sqrt{\mathcal{H}}-\mathcal{H}}$. 


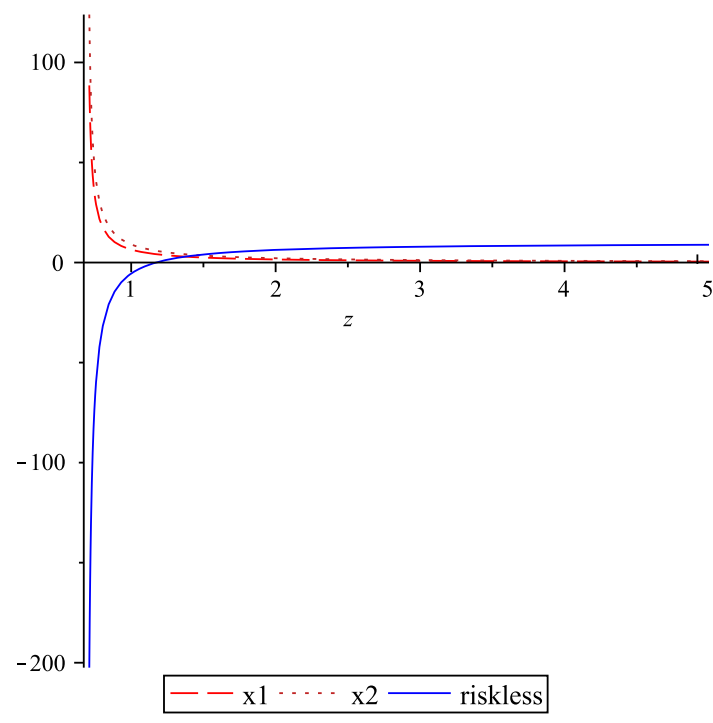

Figure 1. Portfolio positions versus $z_{\alpha}$ for $\mathcal{H}=0.4722, W_{0}=10, R=1.1$, for $b=10.5$.

As in Section 2, for the case $b=W_{0} R$, the optimal portfolio is a totally risk-less portfolio, i.e. all of initial wealth is invested into the risk-less asset in Part A.

The result of Proposition 4 shows the interplay between the two risk parameters acting on the optimal portfolio, namely $z_{\alpha}$ and $\rho$. It is clear that of the two risk control parameters $z_{\alpha}$ and $\rho$ only one can be pushed to high values if one is interested in a meaningful portfolio (that is a feasible portfolio where the chance constraint is active). In Part $\mathrm{C}$, we clearly see that an (MVCCPP) solving investor cannot push the probabilistic protection level beyond the slope of the Capital Market Line, while at the same time aiming for a target larger than the wealth that would be obtained by keeping all initial endowment in the risk-less asset. Part B shows that specifying a high (higher than a specific threshold) variance aversion with any probabilistic guarantee gives an optimal portfolio which disregards the chance constraint. Hence, there is no point in solving (MVCCPP) since the VaR constraint is inactive at the optimal solution.

In Part A case 1, the target value for the wealth $W_{1}$ is chosen less than the critical value $W_{0} R$, hence as in Proposition 1, the probabilistic protection factor $\alpha$ can be pushed to zero (i.e. $z_{\alpha}$ can increase without bound), whereas the variance aversion parameter is limited from above. In case 2 of Part $\mathrm{B}$, the opposite occurs. The target value $b$ is chosen larger than $W_{0} R$, then we only expect a bounded (from above) maximum protection in the chance constraint, which is indeed the case. The maximum protection affordable is $1-\Phi(\sqrt{\mathcal{H}})$, whereas one can now push as much variance aversion as desired into the optimal portfolio.

In Figures 1-3 we illustrate the behaviour of optimal portfolio holdings in case 1 of Part A for $n=2$ with $R=1.1$ and $\bar{\mu}=[0.1,0.05]^{T}$, the expected excess portfolio return (from the risky portion of the portfolio, i.e. $\bar{\mu}^{T} x^{*}$ ) and variance as a function of $z_{\alpha}$ and jointly as $z_{\alpha}$ and $b$ vary, respectively. As expected, as $z_{\alpha}$ increases, $\alpha$ tends to zero, which means a more stringent VaR restriction. Hence, the optimal portfolio tends to shed the initial (for small values of $z_{\alpha}$ ) large long positions in risky assets, and puts increasingly more on the risk-less 


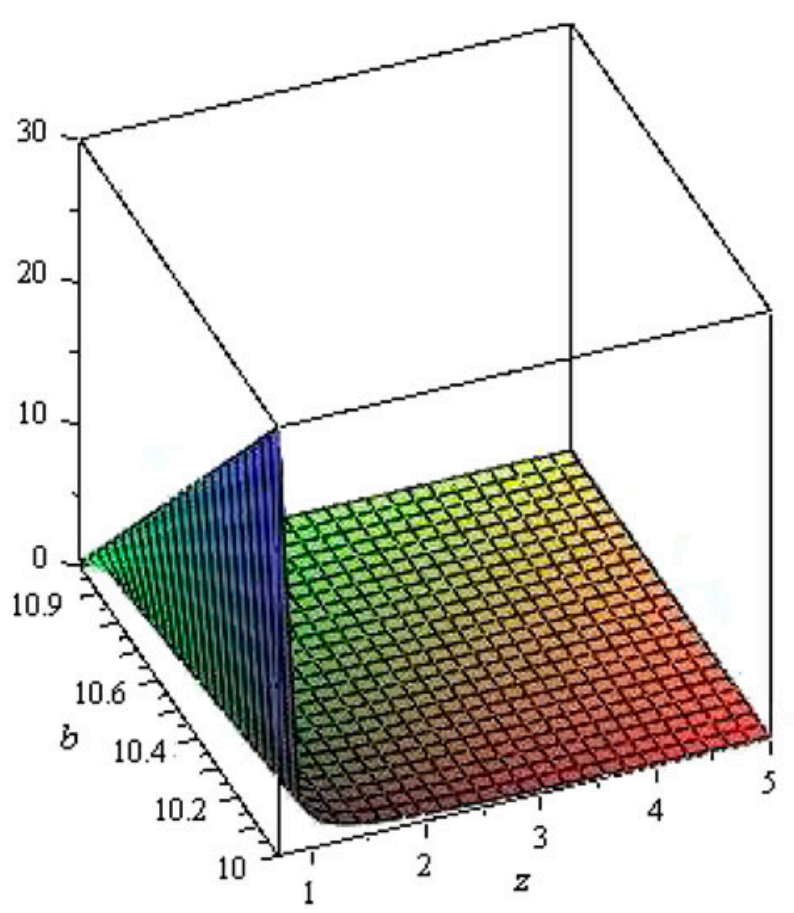

Figure 2. Expected excess return of optimal portfolio versus $z_{\alpha}$ and $b$ for $\mathcal{H}=0.4722, W_{0}=10$, $R=1.1$.

asset. This behaviour is predictable from the optimal portfolio rule in Proposition 1, Part A since the optimal portfolio coefficient $\frac{W_{0} R-b}{z_{\alpha} \sqrt{\mathcal{H}}-\mathcal{H}}$ already makes this relationship transparent. The drop in expected return and variance are quite marked initially as $z_{\alpha}$ and $b$ increase. These remarks apply verbatim to the optimal portfolios of Section 2 as well.

\section{The price of a chance constraint in delegated portfolio management}

In this section, we consider the following problem from Delegated Portfolio Management. $[14,15]$ An investor, who delegates investment of an initial wealth $W_{0}$ to a negative exponential utility portfolio manager, wishes to enforce a probabilistic VaR restriction in the form of a guarantee on the wealth $W_{1}$ realized at the end of the investment horizon.

Typically, in Delegated Portfolio Management, one investigates the form of the optimal contract under certain assumptions on the investor and the portfolio manager using a Principal-Agent framework. In this paper, we assume the form of the contract to be fixed. More precisely, the investor allocates a capital $W_{0}$ to the portfolio manager with the mandate to trade in the set of risky assets and the risk-less asset. The compensation of the manager is a function of the final wealth achieved at the end of the horizon given by

$$
f(W)=A R+\beta W,
$$




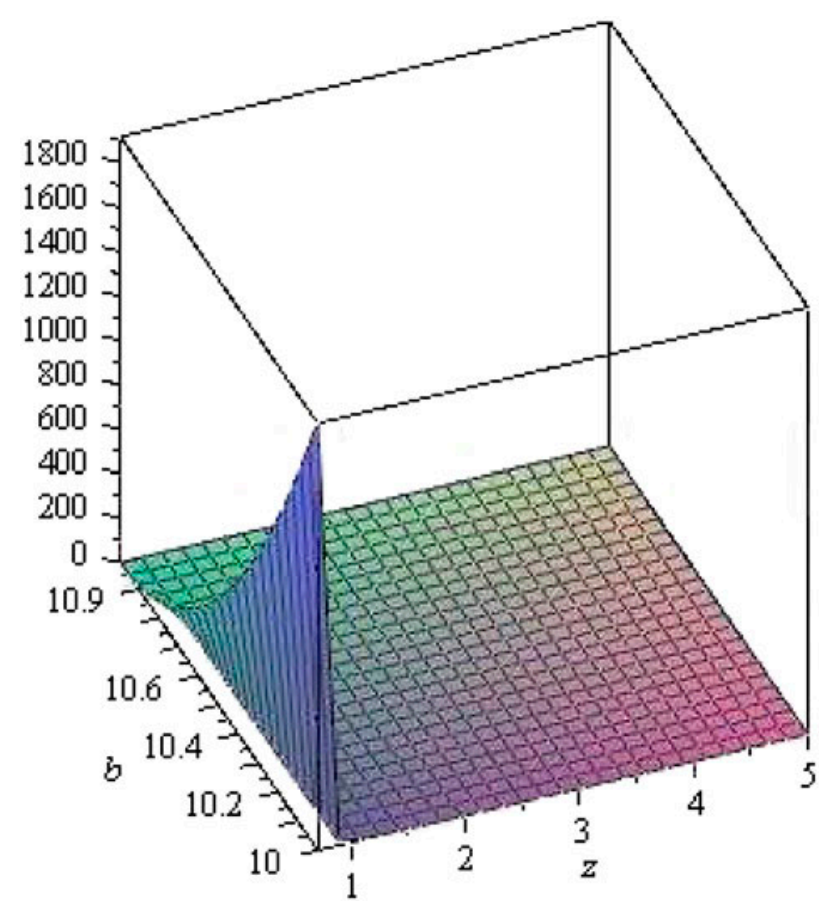

Figure 3. Variance of portfolio return versus $z_{\alpha}$ and $b$ for $\mathcal{H}=0.4722, W_{0}=10, R=1.1$.

where $A$ is a fixed fee received at the beginning of the period and $\beta$ is the fee received on the realized wealth $W$ at the end of the horizon, and given by

$$
W(x)=x^{T} \mathcal{R}+\left[W_{0}-\mathbf{1}^{T} x\right] R .
$$

In the above equation, as in previous sections $x$ is $n$-dimensional vector representing the allocation in the risky assets and $\mathbf{1}$ is a $n$-vector of ones. We assume that the manager can also choose a second contract where the investment is taken on a set of assets where there is no probabilistic restriction, and with pay-off

$$
r\left(W^{\prime}\right)=A R+\beta_{0} W^{\prime}
$$

and final wealth $W^{\prime}$

$$
W^{\prime}(x)=x^{T} \mathcal{R}+\left[W_{0}-\mathbf{1}^{T} x\right] R .
$$

The difference $\Delta=\beta-\beta_{0}$ is the "bonus" of the manager for accepting the investment under the VaR restriction. The purpose of this section is to compute the optimal bonus using the results of the previous section.

The investor wishes to maximize the expected final wealth after rewarding the manager. More precisely, he/she wants to solve

$$
\max _{\Delta} \mathbb{E}[W(\Delta)-f(W(\Delta))]
$$




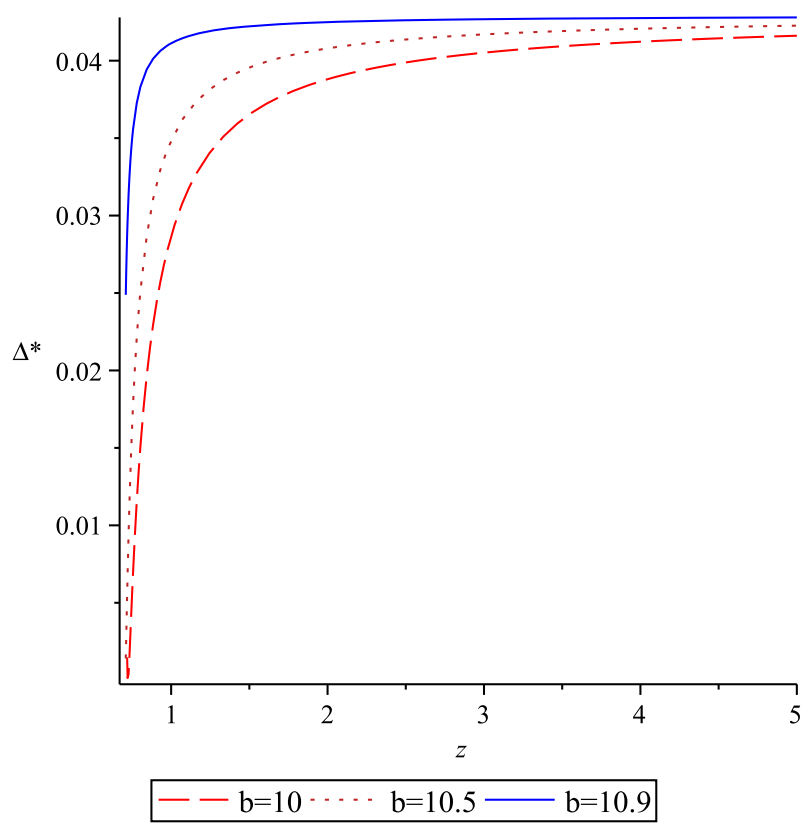

Figure 4. Case 1: $\Delta^{*}$ versus $z_{\alpha}$ for $\mathcal{H}=0.4722, W_{0}=10, R=1.1, \beta_{0}=0.05, \vartheta=0.5$, for $b=10,10.5,10.9$.

where we define

$$
W(\Delta)=\left(x^{*}(\Delta)\right)^{T} \mathcal{R}+\left[W_{0}-\mathbf{1}^{T} x^{*}(\Delta)\right] R
$$

and $x^{*}(\Delta)$ is an VaR-constrained portfolio allocation in the sense that it solves the following problem

$$
\max _{x}\left\{\mathbb{E}\left[-\mathrm{e}^{-\vartheta\left(A R+\left(\beta_{0}+\Delta\right) W(x)\right)}\right]\right\}
$$

where $\vartheta$ is a positive constant, subject to

$$
\operatorname{Pr}\{W(\Delta) \geq b\} \geq 1-\alpha .
$$

Furthermore, $\Delta$ is chosen so that the participation constraint for the manager is satisfied:

$$
\mathbb{E}\left[-\mathrm{e}^{-\vartheta\left(A R+\left(\beta_{0}+\Delta\right) W\left(x^{*}(\Delta)\right)\right)}\right] \geq \mathbb{E}\left[-\mathrm{e}^{-\vartheta\left(A R+\beta_{0} W^{\prime}\right)}\right]
$$

where we define

$$
W^{\prime}=\left(x_{M}\right)^{T} \mathcal{R}+\left[W_{0}-\mathbf{1}_{n}^{T} x_{M}\right] R
$$

and $x_{M}$ solves the problem

$$
\max _{x} \mathbb{E}\left[-\mathrm{e}^{-\vartheta\left(A R+\beta_{0} W^{\prime}\right)}\right] .
$$

The solution $x_{M}$ is known to be [5]

$$
x_{M}=\frac{1}{\vartheta \beta_{0}} \Sigma^{-1}(\mu-R \mathbf{1}) .
$$




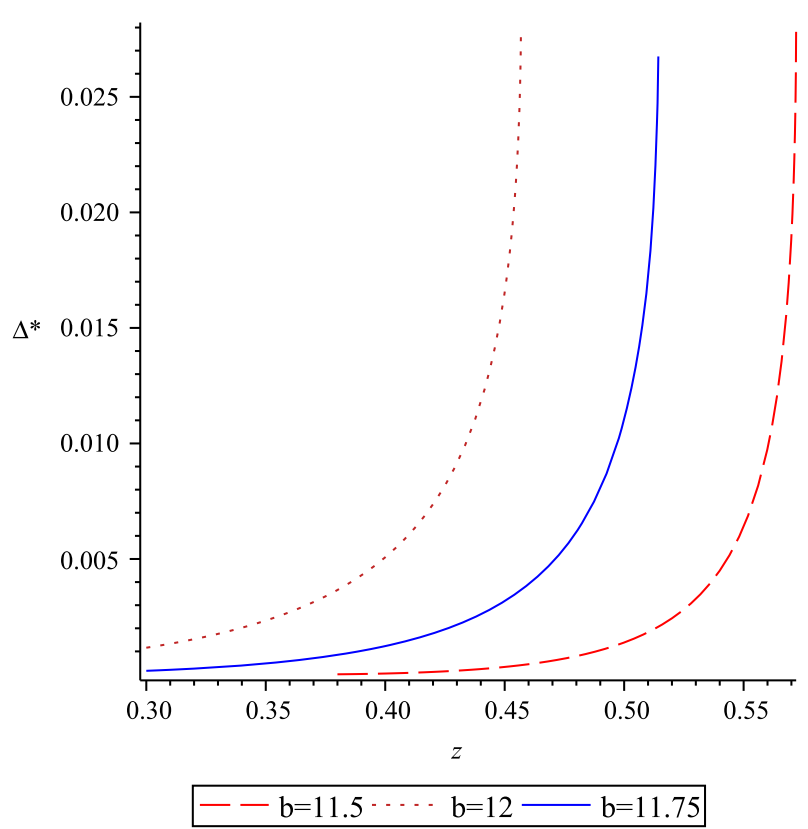

Figure 5. Case 2: $\Delta^{*}$ versus $z_{\alpha}$ for $\mathcal{H}=0.4722, W_{0}=10, R=1.1, \beta_{0}=0.045, \vartheta=10$, for $b=11.5,11.75,12$.

In other words, the manager's reservation utility (on the right hand side of (7)) is measured as its maximum utility that would be attained with a classical Markowitz portfolio ignoring the VaR restriction.

Proposition 5 The solution to the problem (5)-(7), i.e. the price of a chance constraint, is obtained at the smallest of the two conjugate values

$$
\Delta^{*}=\frac{W_{0} R+c \mathcal{H}-\vartheta \beta_{0} c^{2} \mathcal{H} \pm \sqrt{W_{0} R} \sqrt{W_{0} R+2 c \mathcal{H}-2 \vartheta \beta_{0} c^{2} \mathcal{H}}}{\vartheta c^{2} \mathcal{H}}
$$

where $c=\frac{W_{0} R-b}{z_{\alpha} \sqrt{\mathcal{H}}-\mathcal{H}}$ provided that $\vartheta \beta_{0}<\frac{z_{\alpha} \sqrt{\mathcal{H}}-\mathcal{H}}{W_{0} R-b}+\frac{W_{0} R\left(z_{\alpha}-\sqrt{\mathcal{H}}\right)^{2}}{2\left(W_{0} R-b\right)^{2}}$ and $\vartheta\left(\beta_{0}+\Delta^{*}\right)<\frac{1}{c}$ in case 1 or $\vartheta\left(\beta_{0}+\Delta^{*}\right)>\frac{1}{c}$ in case 2 of Part $\mathbf{A}$ of Proposition 4.

One normally expects $\Delta^{*}$ to increase with increasing $z_{\alpha}$, however increasing $z_{\alpha}$ beyond a certain value ceases to be effective since it implies almost zero $\alpha$. Therefore, one expects the increase in $\Delta^{*}$ to follow suit, i.e. to tail off to a limiting value. This kind of behaviour is difficult to infer from the complicated expression (10). However, the tail-off behaviour can be ascertained by taking the limit of the root with the negative sign in front of the square root in (10) (this turns out to be the smaller root), which gives

$$
\frac{1}{2} \frac{\mathcal{H}}{\vartheta W_{0} R}
$$


This limiting bonus depends on the optimal Sharpe ratio $\mathcal{H}$ of the market, and is inversely proportional to risk aversion coefficient $\vartheta$ of the manager and the wealth that would be realized if all endowment were kept in the risk-less asset. This property is also verified by numerical computation. In Figure 4, for case 1 of part $\mathbf{A}$ of Proposition 4 we illustrate the behaviour of the smallest root $\Delta^{*}$ (obtained with the plus sign in front of the square root) for $\mathcal{H}=04722, W_{0}=10, R=1.1, \beta_{0}=0.05, \vartheta=0.5$, and for three different values of $b=10,10.5,10.9$. As expected for larger values of $b$, the the investor pays a larger bonus to the manager, and the bonus increases with increased protection level expressed in increasing $z_{\alpha}$. However, the increase in the bonus in return for more protection is not without bound. It tails off to an upper limit quickly. The limiting value is around 0.043 (also verified from the limit above), which is less than the fixed part $\beta_{0}=0.05$ of the variable portion of the contract.

In Figure 5, we repeat the illustration for case 2 after changing the parameters to fit the conditions of Proposition 5. In this case, the increase in $\Delta^{*}$ is much more abrupt as $z_{\alpha}$ increases, i.e. as more protection through the chance constraint is demanded. At the end, the choice of which of cases 1 and 2 will apply depends on the choice of factors $\alpha, b$ and $\rho$.

\section{Conclusions and outlook}

We conclude with a brief summary of our results. In this paper, we derived closed-form solutions to portfolio selection problems in which short positions are allowed, and with a Value-at-Risk constraint which is a kind of probabilistic chance constraint. For the case of an investor with a risk neutral objective function we showed that if the protection level in the chance constraint is higher than a threshold expressed as the slope of the Capital Market Line and the target wealth is kept below a threshold level (equal to the wealth that would be realized if all endowment was kept in the risk-less account), an optimal portfolio rule is obtained. The result is also extended to multiple periods and yields a myopic portfolio policy which is a replica of the static policy.

When the investor employs a risk-averse mean-variance objective function allowing also control of variance of the portfolio return as well as the VaR constraint, we showed that to obtain an optimal portfolio rule either the probabilistic protection level should be kept under a threshold while the target wealth and variance aversion can be chosen above specific thresholds, or, conversely, the target wealth and aversion to risk should be kept under specific thresholds if one desires a higher protection level. As the protection level increases, the optimal portfolio puts more emphasis on the risk-less asset as expected.

Finally, using our portfolio rule we derived a closed-form expression for the bonus to be paid to a portfolio manager by an investor who desires a VaR type guarantee on the realized wealth. While the resulting expression is complicated, we inferred that the bonus due to the manager for including a VaR constraint increases (as would be expected) with increasing protection level (i.e. decreasing $\alpha$ ) and increasing target wealth if emphasis is placed on the protection level rather than controlling the variance of portfolio return (i.e. part A case 1 of Proposition 4). However, it is interesting that the increase in bonus with respect to $\alpha$ diminishes and tends to zero. That is, pushing for more protection level does not result in increased bonus after a certain value is reached. The limiting bonus depends on the optimal Sharpe ratio of the market, and is inversely proportional to risk aversion coefficient of the manager and the wealth that would be realized if all endowment were kept in the risk-less asset. On the other hand, if emphasis is placed on controlling the variance rather 
than a stringent VaR requirement, the optimal bonus may increase sharply as a function of the protection level although we are dealing here with smaller protection levels compared to case 1 .

It would be interesting to test the Delegated Portfolio Management results of the paper on real financial data by including other instruments like options in the asset universe and relaxing for instance the assumptions of unlimited short sales and borrowing and lending at the same rate. Such a study requires data from portfolio delegation practice and carefully planned experiments, hence will be undertaken in the future.

\section{Acknowledgements}

I am grateful to Stefano Herzel for introducing me to the problem of Delegated Portfolio Management.

\section{References}

[1] Markowitz H. Portfolio selection: efficient diversification of investment. New York: Wiley; 1959.

[2] Steinbach MC. Markowitz revisited: mean-variance models in financial portfolio analysis. SIAM Review. 2001;43:31-85.

[3] Agnew NH, Agnew AR, Rasmussen J, Smith KR. An application of chance constrained programming to portfolio selection in a casualty insurance firm. Manag. Sci. 1969;15: B512-B520.

[4] Charnes A, Cooper WW. Chance-constrained programming. Manag. Sci. 1959;6:73-79.

[5] Ingersoll J. Theory of financial decision making. Maryland: Rowman-Littlefield; 1987.

[6] Pyle DH, Turnovsky SJ. Risk aversion in chance constrained portfolio selection. Manag. Sci. 1971;18:218-225.

[7] Jorion P. Value at risk : the new benchmark for managing financial risk. New York: Wiley; 2007.

[8] Alexander GJ, Baptista AM. A VaR-constrained mean-variance model: implications for portfolio selection and the Basle capital accord, EFA 2001 Barcelona meetings. 2001. Available from: SSRN: http://ssrn.com/abstract=275894 or http://dx.doi.org/10.2139/ssrn.275894.

[9] Alizadeh F, Goldfarb D. Second-order cone programming. Math. Program Ser. B. 2003;95:3-51.

[10] Erdogan E, Iyengar G. An active set method for single-cone second-order cone programs. SIAM J. Optimi. 2006;17:459-484.

[11] Lobo MS, Vandenberghe L, Boyd S, Lebret H. Applications of second-order cone programming. Linear Algebra Appl. 1998;284:193-228.

[12] Ruszczynski A. Nonlinear optimization. Princeton, New Jersey: Princeton University Press; 2007.

[13] Shapiro A, Dentcheva D, Ruszczynski A. Lectures on stochastic programming: modelling and theory. Philadelphia: SIAM-MPS Publications; 2009.

[14] Bhattacharya S, Pfleiderer P. Delegated portfolio management. J. Econ. Theory. 1985;36:1-25.

[15] Fabretti A, Herzel S. Delegated portfolio management with socially responsible investment constraints. Eur. J. Financ. 2012;18:293-309.

[16] Stracca L. Delegated portfolio management: a survey of the theoretical literature. Journal of Economic Surveys. 2006;20:823-848.

[17] Pinar MÇ. Static and dynamic VaR constrained portfolios with application to delegated portfolio management, Technical Report. Bilkent University; 2013. Available from: http://www.ie.bilkent. edu.tr/mustafap/pubs/ccport2rev1.pdf.

[18] Roy AD. Safety first and the holding of assets. Econometrica. 1952;20:431-449.

[19] Başak S, Shapiro A. Value-at-risk based risk management: optimal policies and asset prices. Rev. Financ. Stud. 2001;14:371-405. 
[20] Danielsson J, Jorgensen BN, de Vries CG, Yang X. Optimal portfolio allocation under the probabilistic VaR constraint and incentives for financial innovation. Ann. Financ. 2008;4: 345-367.

[21] Grossman S, Vila JL. Portfolio insurance in complete markets. J. Bus. 1989;62:473-476.

[22] Cornuéjols G, Tütüncü R. Optimization methods in finance. Cambridge: Cambridge University Press; 2007.

[23] Goldfarb D, Iyengar G. Robust portfolio selection problems. Math. Oper. Res. 2003;28:1-38.

[24] Best MJ. Portfolio optimization. Financial Mathematics Series. Boca Raton: Chapman \& Hall/CRC; 2010.

[25] Bertsekas DP. Dynamic programming and stochastic control. New York: Academic Press; 1976. 